\title{
Excitonic magneto-optical Kerr effect in two-dimensional transition metal dichalcogenides induced by spin proximity
}

\author{
J. C. G. Henriques $\odot$ \\ Department and Centre of Physics, and QuantaLab, University of Minho, Campus of Gualtar, 4710-057 Braga, Portugal \\ G. Catarina ${ }^{\circledR}$, A. T. Costa, and J. Fernández-Rossier \\ International Iberian Nanotechnology Laboratory (INL), Avenida Mestre José Veiga, 4715-330 Braga, Portugal \\ N. M. R. Peres $\oplus^{*}$ \\ Department and Centre of Physics, and QuantaLab, University of Minho, Campus of Gualtar, 4710-057 Braga, Portugal \\ and International Iberian Nanotechnology Laboratory (INL), Avenida Mestre José Veiga, 4715-330 Braga, Portugal
}

(Received 8 November 2019; revised manuscript received 11 December 2019; published 8 January 2020)

\begin{abstract}
In this paper, we develop the excitonic theory of the Kerr rotation angle in a two-dimensional (2D) transition metal dichalcogenide at zero magnetic field. The finite Kerr angle is induced by the interplay between spin-orbit splitting and proximity exchange coupling due to the presence of a ferromagnet. We compare the excitonic effect with the single-particle theory approach. We show that the excitonic properties of the 2D material lead to a dramatic change in the frequency dependence of the optical response function. We also find that the excitonic corrections enhance the optical response by a factor of 2 in the case of $\mathrm{MoS}_{2}$ in proximity to a cobalt thin film.
\end{abstract}

DOI: 10.1103/PhysRevB.101.045408

\section{INTRODUCTION}

Proximity effects have been known for decades [1], but their true potential was only unleashed with the rise of twodimensional (2D) materials [2]. When working with bulk materials, proximity effects are negligible, since the size scale of the material is many orders of magnitude superior to the scale along which proximity effects are noticeable, relegating them to localized phenomena occurring within a few nanometers [3]. Working with 2D materials, however, is a strikingly different scenario. Their low dimensionality is responsible for the enhancement of proximity effects. After all, their thickness can be orders of magnitude inferior to the length scale of those effects. This allows the wave function of the material causing the proximity effect to totally engulf the 2D system [4], thus drastically modifying its intrinsic properties. These effects are responsible for inducing new features in the adjacent regions, such as turning a nonmagnetic material into a magnetic one, or giving rise to topologically nontrivial properties where otherwise there were none [3-6].

Among the large variety of $2 \mathrm{D}$ materials, transition metal dichalcogenides (TMDs) are some of the most prominent and studied ones [7]. A monolayer TMD is composed of a layer of transition metal atoms, situated between two layers of chalcogen atoms, forming a trigonal prism structure. A representation of the real lattice of a generic TMD is given in Fig. 1. Contrary to graphene, the existence of different types of atoms in each sublattice leads to the opening of gaps at the corners of the first Brillouin zone. According to Ref. [8], these

*peres@ fisica.uminho.pt, nuno.peres@inl.int band gaps are of the order of $1 \mathrm{eV}$. Another aspect in which TMDs differ from graphene is the existence of strong spinorbit coupling (SOC), due to the presence of heavy atoms with $d$ orbitals. To describe the band structure of these materials, one uses a massive Dirac Hamiltonian, to which a SOC term must be added. The existence of spin-orbit coupling is also responsible for the coupling of spin and valley, which leads to valley selective helicity in interband transitions [9]. A key aspect to control the valley degrees of freedom is to break the existing symmetry between the point $K$ and $K^{\prime}$ at the corners of the first Brillouin zone. Unfortunately, this proves to be an extremely hard task when using an external magnetic field, since fields as large as $\sim 10 \mathrm{~T}$ are necessary to produce a minute splitting of $\sim 1 \mathrm{meV}$ [10-15].

It is at this point that a proximitized TMD becomes an entirely new system with the desired properties, since it has been shown that proximity to a magnetic material produces the needed valley splitting magnitudes [16]. Previous works have already studied the valley manipulation due to proximity to antiferromagnetic $[17,18]$ and ferromagnetic $[13,16,19]$ substrates, as well as the effect of proximity to $\mathrm{CrI}_{3}$, an ultrathin ferromagnetic semiconductor $[14,15,20]$.

With the ability to control both the spin and valley degrees of freedom by ingeniously choosing an adequate substrate, we can explore magneto-optical effects, such as the Kerr rotation angle in the absence of magnetic fields. Although these kind of effects are vastly studied and used in bulk materials [22-24], their true potential in 2D materials is yet to be fulfilled. In this paper, we discuss the effects that proximity-induced phenomena have in the band structure and optical conductivity (both longitudinal and Hall) of a TMD. We show that the Hall conductivity becomes nonzero when the valley symmetry is 


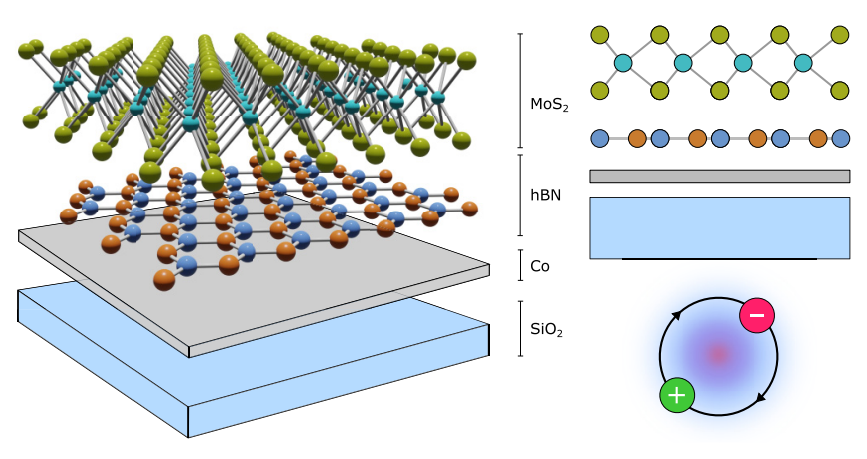

FIG. 1. Representation of the system considered in this paper: a heterostructure composed of $\mathrm{MoS}_{2} / \mathrm{hBN} /$ cobalt thin film/quartz; both a three-dimensional perspective and a transverse cut view of the heterostructure are shown. The hBN is single layer and acts as a buffer layer to protect $\mathrm{MoS}_{2}$ from direct contact with the metallic cobalt thin film (three layers). An artistic view of an exciton formed in $\mathrm{MoS}_{2}$ by the impinging electromagnetic radiation is also depicted. The band structure of this heterostructure was first discussed in Ref. [21].

broken due to proximity to a magnetic thin film. The finite Hall conductivity is the key feature to obtain a nonzero Kerr rotation angle.

This paper is organized as follows: We start in Sec. II by introducing the model Hamiltonian that will be used throughout the text, and apply it to the case of $\mathrm{MoS}_{2}$ on a heterostructure composed of $\mathrm{MoS}_{2}$ /hexagonal boron nitride (hBN) single layer/cobalt thin film/quartz. Afterwards, in Sec. III, we compute the absorbed power of a 2D material from Fermi's golden rule, for both linearly and circularly polarized light, and establish its relation with different entries of the conductivity tensor. Finally, in Sec. IV, we apply this formalism to the study of the Hall conductivity, and later to the Kerr angle, in the presence of excitonic effects. An Appendix gives the transformation of the Bethe-Salpeter equation to real space.

\section{MODEL HAMILTONIAN AND BAND STRUCTURE}

In this section, we introduce the model Hamiltonian that will be used throughout the text, composed of the standard Dirac Hamiltonian with finite mass to which a spin-orbit contribution and an exchange term are added (a numerical value of the latter has been determined from $a b$ initio calculations). We will be specific and consider the case of a monolayer $\mathrm{MoS}_{2}$ heterostructure (see Fig. 1) composed of $\mathrm{MoS}_{2} / \mathrm{hBN}$ single layer/cobalt thin film/quartz, a system for which a giant magnetic exchange was found [21]. Following that recent work [21], the cobalt thin film is composed of the three layers. The hBN single layer is used as a buffer layer and the cobalt provides the proximity-induced exchange. Since both the cobalt and the hBN are extremely thin, we assume that most of the screening of the electric field between the electron and the hole is provided by the quartz (or otherwise) substrate.

\section{A. Model}

To describe our system we adopt a low-energy effective Hamiltonian with the structure $H=H_{0}+H_{\text {SOC }}+H_{\text {ex }}$, where
$H_{0}$ is the usual Dirac Hamiltonian, $H_{\text {SOC }}$ describes the spin orbit coupling, and $H_{\mathrm{ex}}$ characterizes the exchange splitting due to magnetic proximity effects. When written explicitly, and in agreement with Refs. $[9,16,25,26]$, the total Hamiltonian is given by

$$
\begin{aligned}
H= & v_{F} \hbar\left(\tau k_{x} \sigma_{x}+k_{y} \sigma_{y}\right)+\frac{m}{2} \sigma_{z} \\
& +\tau s_{z}\left(\lambda_{c} \frac{1+\sigma_{z}}{2}+\lambda_{v} \frac{1-\sigma_{z}}{2}\right) \\
& -s_{z}\left(B_{c} \frac{1+\sigma_{z}}{2}+B_{v} \frac{1-\sigma_{z}}{2}\right),
\end{aligned}
$$

where $v_{F}$ is the Fermi velocity; $\hbar$ is the reduced Planck's constant; $\tau= \pm 1$ is the valley index referring to the $K$ and $K^{\prime}$ valleys, respectively; $s_{z}= \pm 1$ is the spin index labeling spin up and spin down, respectively; $\sigma_{x}, \sigma_{y}, \sigma_{z}$ are $2 \times 2$ Pauli matrices; $k_{x}$ and $k_{y}$ are the $x$ and $y$ components of the wave vector $\mathbf{k} ; m$ is the band gap when no other contributions are considered; $\lambda_{c}$ and $\lambda_{v}$ characterize the spin-orbit coupling splitting in the conduction and valence band; and $B_{c}$ and $B_{v}$ describe the effective exchange splitting for the conduction and valence band, respectively, induced by proximity (these parameters can be determined using $a b$ initio methods, as noted before).

Solving the eigenproblem $H\left|u_{\alpha}^{\tau, s_{z}}\right\rangle=E_{\alpha}\left|u_{\alpha}^{\tau, s_{z}}\right\rangle$, with $\alpha=$ $\{c, v\}$ (or $\alpha= \pm 1$, respectively, depending on the context), one easily obtains

$$
\left|u_{c}^{\tau, s_{z}}\right\rangle=\left(\begin{array}{c}
\frac{\mathcal{M}_{c}^{\tau, s_{z}}}{\sqrt{\left(\mathcal{M}_{c}^{\tau, s_{z}}\right)^{2}+v_{F}^{2} \hbar^{2} k^{2}}} \\
\frac{\tau v_{F} \hbar k e^{\tau i \theta}}{\sqrt{\left(\mathcal{M}_{c}^{\tau, s_{z}}\right)^{2}+v_{F}^{2} \hbar^{2} k^{2}}}
\end{array}\right),
$$

and

$$
\left|u_{v}^{\tau, s_{z}}\right\rangle=\left(\begin{array}{c}
\frac{-\tau v_{F} \hbar k e^{-\tau i \theta}}{\sqrt{\left(\mathcal{M}_{v}^{\tau, s}\right)^{2}+v_{F}^{2} \hbar^{2} k^{2}}} \\
\frac{\mathcal{M}_{v}^{\tau, s}}{\sqrt{\left(\mathcal{M}_{v}^{\tau, s}\right)^{2}+v_{F}^{2} \hbar^{2} k^{2}}}
\end{array}\right),
$$

with $\tan \theta=k_{y} / k_{x}, \mathcal{M}_{c}^{\tau, s_{z}}=m / 2+B_{v} s_{z}-\lambda_{v} s_{z} \tau+E_{c}$, and $\mathcal{M}_{v}^{\tau, s_{z}}=m / 2-B_{c} s_{z}+\lambda_{c} s_{z} \tau-E_{v}$, considering

$$
E_{\alpha}=s_{z}\left[\left(\lambda_{c}+\lambda_{v}\right) \tau-\left(B_{c}+B_{v}\right)\right]+\alpha \sqrt{\left(\frac{\zeta_{\tau, s_{z}}}{2}\right)^{2}+v_{F}^{2} \hbar^{2} k^{2}},
$$

where $\zeta_{\tau, s_{z}}=m+s_{z}\left(B_{v}-B_{c}+\lambda_{c} \tau-\lambda_{v} \tau\right)$ is the gap between the valence and conduction band for a specific choice of $\tau$ and $s_{z}$.

The total electronic Hamiltonian is the sum of $H$ with the Rytova-Keldysh potential, defined as [27,28]

$$
V(r)=\frac{e^{2}}{4 \pi \epsilon_{0}} \frac{\pi}{2} \frac{1}{r_{0}}\left[\mathbf{H}_{0}\left(\frac{\kappa r}{r_{0}}\right)-Y_{0}\left(\frac{\kappa r}{r_{0}}\right)\right],
$$

where $r_{0} \sim d \epsilon / 2$, with $d$ and $\epsilon$ the thickness and dielectric function of the 2D material, respectively (microscopically, $r_{0}$ relates to the polarizability of the $2 \mathrm{D}$ system); $\kappa$ is the mean dielectric function of the media surrounding the 2D material; $\epsilon_{0}$ is the vacuum permittivity; $e$ is the elementary charge; and $\mathbf{H}_{0}(x)$ is the Struve function, and $Y_{0}(x)$ is the Bessel function 
TABLE I. Parameters used throughout the text for the Hamiltonian of $\mathrm{MoS}_{2}$ near a cobalt thin film. Besides the parameters shown, we also considered an effective mass $m^{*}=0.5 m_{0}$, with $m_{0}$ the bare electron mass, and a mean dielectric constant of the substrate (quartz) and capping layer (vacuum) of $\kappa=2.45$. Although these last parameters do not appear in the independent-particle Hamiltonian, they are necessary to compute the binding energies of the excitons in the considered apparatus, as they appear in the interaction potential energy. All the parameters, except the effective mass, were taken from Ref. [21], where a giant exchange for $\mathrm{MoS}_{2}$ on cobalt (three layers) was found. The value of the effective mass used was taken from Ref. [29] for $\mathrm{MoS}_{2}$.

\begin{tabular}{lccc}
\hline \hline Variable & Value & Variable & Value \\
\hline$m$ & $1.759 \mathrm{eV}$ & $B_{c}$ & $1.964 \mathrm{meV}$ \\
$\lambda_{c}$ & $-1.361 \mathrm{meV}$ & $B_{v}$ & $6.365 \mathrm{meV}$ \\
$\lambda_{v}$ & $72.96 \mathrm{meV}$ & $v_{F} \hbar$ & $3.52 \mathrm{eV} \mathrm{\AA}$ \\
\hline \hline
\end{tabular}

of the second kind. This potential is the solution of the Poisson equation for a thin film embedded in a medium.

\section{B. Band structure of the heterostructure of $\mathrm{MoS}_{2}$}

Using the energy spectrum obtained in Eq. (4), and the realistic parameters of Table I, we plot in Fig. 2 the valence and conduction bands in the vicinity of the $K$ and $K^{\prime}$ valleys, for both spin-up and spin-down states. The figure is composed of two distinct cases: when only spin-orbit coupling is considered (top row); and when both spin-orbit coupling and proximity-induced exchange splitting are included (bottom row). Studying the top row, we see that we no longer have the two degenerate spin bands characteristic of a Dirac Hamiltonian, since the presence of spin-orbit coupling breaks the symmetry between the two different spin states. This effect lifts the spin degeneracy and unfolds each band in two. The energy difference between spin-up and spin-down states is $\Delta_{\text {spin }}^{c}=$ $2 \lambda_{c}$ for the conduction band, and $\Delta_{\text {spin }}^{v}=2 \lambda_{v}$ for the valence band. Comparing the bands of the $K$ and $K^{\prime}$ valleys, we observe that, although the bands associated with spin up and spin down are switched, the valleys have a symmetric band structure, that is, only the role of the spins is interchanged between the two valleys. This is a consequence of time-reversal symmetry. Studying the bottom row, we realize that bands that were once aligned are now shifted relative to each other due to the exchange interaction induced by the magnetic proximity effect. The relative shifts are $\Delta_{\text {valley }}^{c}=2 B_{c}$ for the conduction band, and $\Delta_{\text {valley }}^{v}=2 B_{v}$ for the valence band. The presence of this proximity effect is thus responsible for breaking the valley symmetry, which leads to a quite different interaction of the TMD with the two types of circularly polarized light. Consequently, this disparity in the interaction with both kinds of circularly polarized light allows us to exploit some material properties that were otherwise inaccessible, such as a finite optical Hall conductivity, in the absence of a magnetic field.

We next present in Table II the gaps associated with every considered transition, in the case where both spin-orbit coupling and exchange splitting are considered (introduced before as $\zeta_{\tau, s_{z}}$ ). These values will be useful for future reference. Analyzing the presented data, one quickly realizes

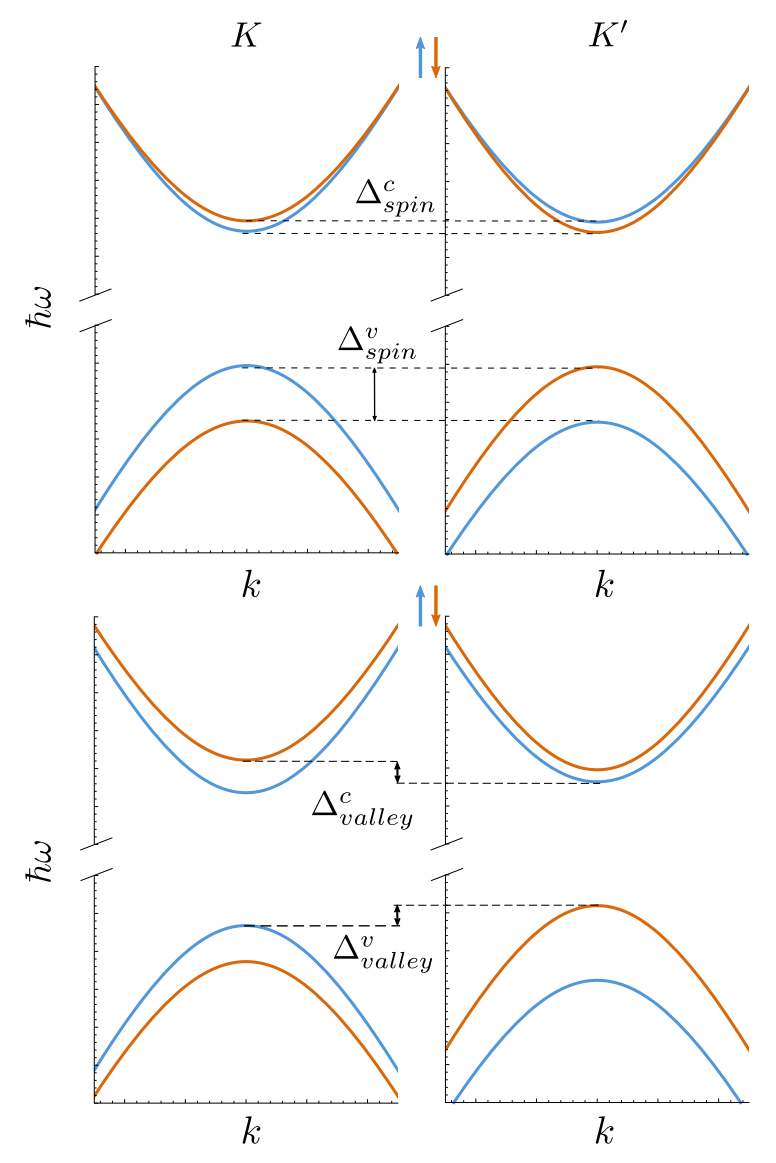

FIG. 2. Band structure near the Dirac cones for $\mathrm{MoS}_{2}$ near a cobalt thin film, using Eq. (4), and the parameters of Table I. To allow an easier visualization of the different gaps, the parameters of Table I were changed to $15 B_{c}, 5 B_{v}$, and $10 \lambda_{c}$. These changes make the gaps more perceptible, while keeping their relative order, as well as the relative magnitude between the parameters, the same. The figure is structured as follows: The left plots refer to the $K$ valley, while the right ones to the $K^{\prime}$ valley; the top row was plotted considering only spin-orbit coupling effects, while the bottom row was plotted with both spin-orbit coupling and exchange splitting considered. All plots are presented with the same scale, with the energies $\hbar \omega$ and wave vector $k$ given in arbitrary units. Looking at the top row, we can see that the presence of spin-orbit coupling lifts the spin degeneracy, and splits each band into two. The bands of spin-up and spin-down states are split by $\Delta_{\text {spin }}^{c / v}=2 \lambda_{c / v}$ for both valleys. The only difference between the two valleys is the swap of the spin-up and spin-down bands. Studying the bottom row, we realize that the presence of exchange splitting breaks the valley symmetry, and the bands that were previously aligned are now shifted by $\Delta_{\text {valley }}^{c / v}=2 B_{c / v}$.

that, as expected, in the absence of exchange splitting, that is, when $B_{c}=B_{v}=0$, the two valleys are again equivalent in terms of gap values. Furthermore, it becomes clear that it is the difference $\left(B_{c}-B_{v}\right)$ that actually controls the valley asymmetry.

\section{OPTICAL CONDUCTIVITY}

In this section, we obtain the absorbed power, from Fermi's golden rule, for both linearly and circularly polarized light. 
TABLE II. Analytical expressions and numerical value for the gaps of the transitions $\left(\tau, s_{z}, v\right) \rightarrow\left(\tau, s_{z}, c\right)$, that is, the transition between the valence and conduction band, for a state of spin projection $s_{z}$ in the valley $\tau$ for $\mathrm{MoS}_{2}$. Working in the limit of vertical transitions we ignore transitions from the valley $\tau$ to $-\tau$. No spin flips are considered. The numerical values were obtained using Table I. This table emphasizes that the asymmetry between the two valleys (seen on the bottom row of Fig. 2) is governed by the difference between $B_{c}$ and $B_{v}$.

\begin{tabular}{lcc}
\hline \hline$\left(\tau, s_{z}, v\right) \rightarrow\left(\tau, s_{z}, c\right)$ & Expression $\zeta_{\tau, s_{z}}$ & Value $(\mathrm{eV})$ \\
\hline$\tau=1, s_{z}=1$ & $m+\lambda_{c}-\lambda_{v}-B_{c}+B_{v}$ & 1.689 \\
$\tau=1, s_{z}=-1$ & $m-\lambda_{c}+\lambda_{v}+B_{c}-B_{v}$ & 1.829 \\
$\tau=-1, s_{z}=1$ & $m-\lambda_{c}+\lambda_{v}-B_{c}+B_{v}$ & 1.838 \\
$\tau=-1, s_{z}=-1$ & $m+\lambda_{c}-\lambda_{v}+B_{c}-B_{v}$ & 1.680 \\
\hline \hline
\end{tabular}

Afterwards, we establish the relation between the absorbed power and the conductivity tensor. The ultimate goal is the calculation of the optical conductivity in both the noninteracting (see Supplemental Material [30]) and interacting limits for both linearly and circularly polarized light.

For the sake of clarity, a comment on notation is now in order. When referring to circularly polarized light, we will label the two orthogonal components as $\tilde{\sigma}_{ \pm}$. Later in this paper we will define conductivities also labeled by the greek letter $\sigma$, namely, $\sigma_{ \pm}$. To avoid confusion between conductivity and polarization, it should be clear that when a tilde $(\sim)$ is used, we are labeling a polarization component, and when it is not we are referring to conductivities.

\section{A. Fermi's golden rule and absorbed power}

Let us start defining the light's electric field as

$$
\mathbf{E}=\frac{1}{2}\left(\mathbf{E}_{0} e^{i \omega t}+\mathbf{E}_{0}^{*} e^{-i \omega t}\right),
$$

and work in the dipole approximation, such that the interaction between light and matter is given by $H_{\text {int }}=-e \mathbf{r} \cdot \mathbf{E}$.

From Fermi's golden rule, one obtains the transition rate between two different states. If we multiply the transition rate by the energy associated with the said transition, and sum over all initial and final states, we obtain the total power absorbed by our system, which is given by

$$
\begin{aligned}
P= & \frac{2 \pi}{\hbar} \frac{1}{4} \sum_{i, f}\left|\hbar \omega_{i f}\right|\left|\left\langle f\left|e \mathbf{r} \cdot \mathbf{E}_{0}\right| i\right\rangle\right|^{2} \\
& \times\left[\delta\left(\hbar \omega-\hbar \omega_{i f}\right)+\delta\left(\hbar \omega+\hbar \omega_{i f}\right)\right],
\end{aligned}
$$

where $P$ is the power absorbed; the sum is made over all initial (i) and final $(f)$ states; and $\hbar \omega_{i f}=E_{i}-E_{f}$ is the difference between the initial and final state energies. Equation (7) is valid for systems with mean occupation numbers 1 and 0 for the initial and final states, respectively, such as, for example, the transitions between a full valence band and an empty conduction band. If this is not the case, one must add a term which takes into account the mean occupation of the states. Although we present two delta functions in all equations, we will only work with positive frequencies, making one of the delta functions redundant.
Let us now consider the case of linearly polarized light. The electric field's amplitude for this type of polarization is $\mathbf{E}_{0}=E_{0} \hat{u}_{x}$, with $\hat{u}_{x}$ the unit vector of the $x$ axis. Thus, the absorbed power is given by

$$
\begin{aligned}
P_{x}= & \frac{\pi}{2 \hbar} e^{2} E_{0}^{2} \sum_{i, f}\left|\hbar \omega_{i f}\right||\langle f|x| i\rangle|^{2} \\
& \times\left[\delta\left(\hbar \omega-\hbar \omega_{i f}\right)+\delta\left(\hbar \omega+\hbar \omega_{i f}\right)\right] .
\end{aligned}
$$

For circularly polarized light the amplitude of the electric field is $\mathbf{E}_{0}=E_{0}\left(\hat{u}_{x} \pm i \hat{u}_{y}\right) / \sqrt{2}$, for $\tilde{\sigma}_{ \pm}$polarization, and the absorbed power is

$$
\begin{aligned}
P_{ \pm}= & \frac{\pi}{4 \hbar} e^{2} E_{0}^{2} \sum_{i, f}\left|\hbar \omega_{i f}\right||\langle f|x \pm i y| i\rangle|^{2} \\
& \times\left[\delta\left(\hbar \omega-\hbar \omega_{i f}\right)+\delta\left(\hbar \omega+\hbar \omega_{i f}\right)\right],
\end{aligned}
$$

with $\hat{u}_{y}$ the unit vector of the $y$ axis.

\section{B. Relation between the absorbed power and the conductivity}

Now that the expressions for the power are determined, we want to establish their relation with the conductivity. To this end we define the current density vector as

$$
\mathbf{J}=\frac{1}{2}\left(\mathbf{J}_{0} e^{i \omega t}+\mathbf{J}_{0}^{*} e^{-i \omega t}\right)
$$

with $\mathbf{J}_{0}=\overline{\bar{\sigma}} \mathbf{E}_{0}$, where $\overline{\bar{\sigma}}$ is the conductivity tensor given by (for an isotropic system)

$$
\overline{\bar{\sigma}}=\left(\begin{array}{cc}
\sigma_{x x 1}+i \sigma_{x x 2} & \sigma_{x y 1}+i \sigma_{x y 2} \\
-\sigma_{x y 1}-i \sigma_{x y 2} & \sigma_{x x 1}+i \sigma_{x x 2}
\end{array}\right),
$$

where $\sigma_{x x}$ is the longitudinal conductivity, $\sigma_{x y}$ is the Hall conductivity, and the indices 1 and 2 refer to the real and imaginary parts, respectively. We can now take advantage of these two expressions to calculate the power absorbed in a different way. To this end, we need to integrate over the area $A$ of our material the dot product $\mathbf{J} \cdot \mathbf{E}$, and take the average over one period $T$. A simplified way of writing this is

$$
P=\langle P(t)\rangle_{T}=\frac{1}{2} \int_{A} d A \operatorname{Re}\left(\mathbf{J}_{\mathbf{0}}^{*} \cdot \mathbf{E}_{\mathbf{0}}\right),
$$

with $\mathbf{E}_{0}$ and $\mathbf{J}_{0}$ defined in agreement with Eqs. (6) and (10). One can now use this expression to establish the relation between the absorbed power and different elements of the conductivity tensor $\overline{\bar{\sigma}}$.

For linearly polarized light, with $\mathbf{E}_{0}=E_{0} \hat{u}_{x}$, one can easily obtain

$$
\sigma_{x x 1}=\frac{2 P_{x}}{A E_{0}^{2}},
$$

which gives a direct relation between the power absorbed with linearly polarized light and the real part of the longitudinal conductivity.

For circularly polarized light, with $\mathbf{E}_{0}=E_{0}\left(\hat{u}_{x} \pm i \hat{u}_{y}\right) / \sqrt{2}$, one obtains

$$
\begin{aligned}
\sigma_{x x 1} & =\frac{P_{+}+P_{-}}{A E_{0}^{2}}, \\
\sigma_{x y 2} & =\frac{P_{-}-P_{+}}{A E_{0}^{2}} .
\end{aligned}
$$


Now that everything is set up, we can start the explicit calculation of the optical conductivity. For the noninteracting limit this is done in the Supplemental Material [30].

\section{EXCITONIC EFFECTS IN THE KERR ANGLE}

Up to this point we have considered a general approach. From now on we will build on what has already been done for the noninteracting case (see Supplemental Material [30]) and expand it to the interacting case, where excitonic effects will be considered (see the Appendix for further information on the solution of the excitonic problem). In this section, we use the formalism developed in the Appendix for solving the 2D Wannier equation, where we show how it can be obtained from the Bethe-Salpeter equation (see also Ref. [31]). Afterwards, we will use a semianalytical method to compute the longitudinal and Hall optical conductivities considering excitonic effects, for both linearly and circularly polarized light. Finally, we study the effect of excitons on the Kerr rotation angle and show that it is larger than that for thin cobalt films by about one order of magnitude.

Continuing the work presented in Sec. III, we write the conductivities [31] $\sigma_{x x}, \sigma_{+}$, and $\sigma_{-}$in the general form

$$
\begin{aligned}
\frac{\sigma_{\mu}^{\tau, s_{z}}}{\sigma_{0}}= & 4 i \sum_{\nu}\left|\zeta_{\tau, s_{z}}+E_{v}\right| \Lambda_{\nu}^{\mu}\left(\frac{1}{\hbar \omega-\zeta_{\tau, s_{z}}-E_{\nu}+i \eta}\right. \\
& \left.+\frac{1}{\hbar \omega+\zeta_{\tau, s_{z}}+E_{\nu}+i \eta}\right)
\end{aligned}
$$

with $\mu=\{x x ;+;-\} ; \zeta_{\tau, s_{z}}=m+s_{z}\left(-B_{c}+B_{v}+\lambda_{c} \tau-\lambda_{v} \tau\right)$ is the gap between the valence and conduction band for a given combination of valley $\tau$ and spin $s_{z}$; and $E_{v}$ is the exciton energy level associated with the quantum number $v$ (including both the principal and magnetic quantum numbers). Only three magnetic quantum numbers produce a nonzero result, $m=0$ and $m= \pm 2$, the largest contribution being, by far, that of $m=0 ; \eta$ is the nonradiative decay rate, encompassing all possible decay channels; finally, the element that differs depending on the desired conductivity is $\Lambda_{v}^{\mu}$, defined as $A\left|\left\langle v, Q\left|\mathbf{r} \cdot \hat{\mathbf{e}}_{\mu}\right| \mathrm{GS}\right\rangle\right|^{2}$, with $A$ the area of the $2 \mathrm{D}$ material, and $\mathbf{r} \cdot \hat{\mathbf{e}}_{\mu}$ equal to $x,(x+i y) / \sqrt{2}$, and $(x-i y) / \sqrt{2}$, for the cases of $\sigma_{x x}, \sigma_{+}$, and $\sigma_{-}$, respectively. The ket $|\nu, Q\rangle$ is defined as

$$
|v, Q\rangle=\frac{1}{\sqrt{A}} \sum_{\mathbf{k}} \phi_{\nu}(\mathbf{k}) a_{\mathbf{k}+\mathbf{Q}, c}^{\dagger} a_{\mathbf{k}, v}|\mathrm{GS}\rangle
$$

where $|\mathrm{GS}\rangle$ stands for the electronic ground state of the TMD, that is, a filled valence band and an empty conduction band (for more details, see the Appendix). The matrix element is promptly computed writing the position operator in second quantization.

To compute the exciton binding energy of $\mathrm{MoS}_{2}$ on a substrate of quartz in the vicinity of a thin cobalt film, we used a mean dielectric constant $\kappa=2.45$ and a screening parameter $r_{0}=41.4 \AA$ (also, for the numerical solution of the Wannier equation, we have used $\Omega_{\min }=-1, \Omega_{\max }=5$, $N=100$, and $A=6$; see the Appendix for the method of solving the Wannier equation). A binding energy of $0.316 \mathrm{eV}$ was found for the exciton. Inserting the computed binding

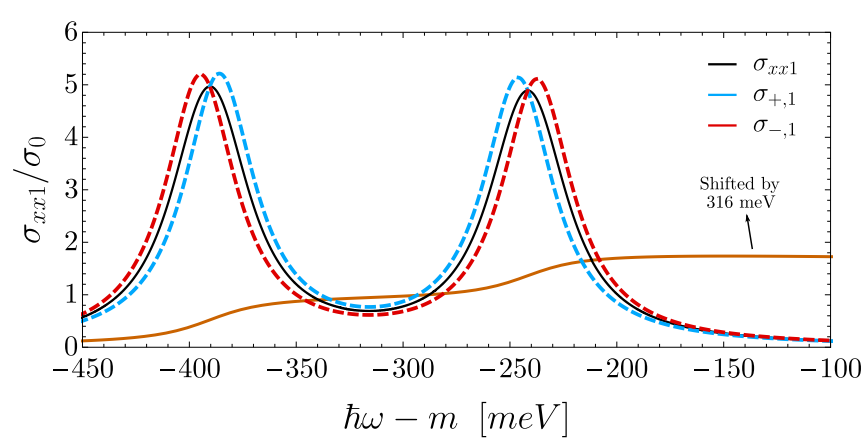

FIG. 3. Representation of $\sigma_{x x 1}, \sigma_{+, 1}$, and $\sigma_{-, 1}$, defined as in Eq. (16). It is easy to see that, as expected, $\sigma_{x x 1}$ can be obtained from the mean of $\sigma_{+, 1}$ and $\sigma_{-, 1}$. The linear conductivity in the noninteracting limit is also shown. It should be noted that the noninteracting line was shifted by $316 \mathrm{meV}$ (the exciton's binding energy) in order to bring all the plots to the same spectral region. Comparing them, one realizes that their line shape is severely modified by the presence of excitons. It is also worth noting that the large splitting $(\sim 140 \mathrm{meV})$ between the two sets of peaks is due to spin-orbit coupling effects, while the small splitting $(\sim 9 \mathrm{meV})$ is a consequence of the valley asymmetry induced by proximity. One final remark should be made: While constructing the plots for $\sigma_{+, 1}$ and $\sigma_{-, 1}$, the absence of an interaction of $\sigma_{+, 1}\left(\sigma_{-, 1}\right)$ with the $K^{\prime}(K)$ valley was observed. Only excitonic peaks with quantum numbers $n=1$, and $m=0$ were considered. All conductivities are presented in units of graphene universal conductivity $\sigma_{0}=e^{2} / 4 \hbar$. The variables of Table I were used, and a nonradiative decay rate $\eta=20 \mathrm{meV}$ was considered. The precise energies where the excitonic resonances appear are presented in Table III. Similar results have been reported in Ref. [26].

energy into Eq. (16), and taking the real part only, one obtains the plots presented in Fig. 3.

These plots show an interesting behavior, as the presence of excitons produces major changes in the conductivities' line shape relative to the noninteracting limit. Indeed, we can also divide the figure in two sets of peaks separated by approximately $140 \mathrm{meV}$. This large splitting between the two sets of peaks is a direct consequence of spin-orbit coupling, while the small splitting within each set is due to the breaking of valley symmetry induced by proximity. Therefore, an experiment measuring the absorption of circularly polarized electromagnetic radiation will be able to unveil the value of the exchange interaction. Using the information of Table III, one realizes that, when only excitons with $n=1$ and $m=0$

TABLE III. Energies at which the excitonic resonances appear in Fig. 3. The data presented emphasize how $\sigma_{+, 1}$ and $\sigma_{-, 1}$ are valley selective, since the excitonic resonances of $\sigma_{+}$are associated with the $K$ valley $(\tau=1)$, and the ones of $\sigma_{-}$are associated with $K^{\prime}$ ( $\tau=$ $-1)$. The origin of the different splittings of the excitonic peaks is also made clear.

\begin{tabular}{lrrc}
\hline \hline & $\tau$ & $s_{z}$ & $E_{g}^{\tau, s_{z}}+E_{v}-m$ \\
\hline$\sigma_{+}$ & 1 & 1 & $-386 \mathrm{meV}$ \\
& 1 & -1 & $-246 \mathrm{meV}$ \\
$\sigma_{-}$ & -1 & 1 & $-237 \mathrm{meV}$ \\
& -1 & -1 & $-395 \mathrm{meV}$ \\
\hline \hline
\end{tabular}




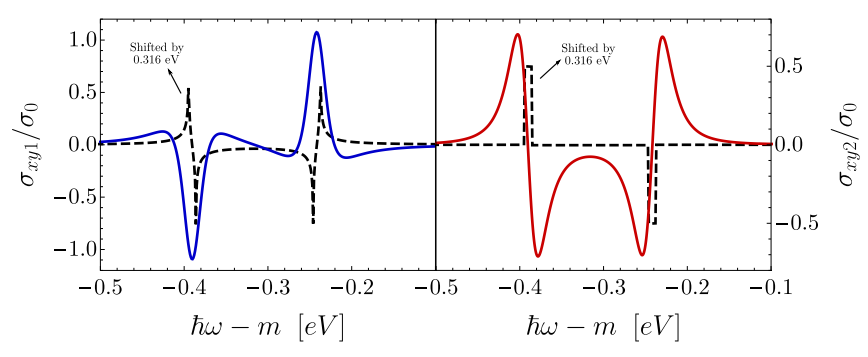

FIG. 4. Representation of both real (left panel) and imaginary (right panel) parts of the Hall conductivity, obtained from Eq. (18). When we compare these plots with the ones for the noninteracting theory (represented by the dashed lines), we realize, quite unexpectedly, that the presence of excitonic effects induces a swap of behavior between the real and imaginary parts of the Hall conductivity, while keeping a similar magnitude. We should note that, similarly to what was done in Fig. 3, the plots of the noninteracting limit were shifted by $0.316 \mathrm{eV}$, in order to show all the plots in the same energy range. All the conductivities are presented in units of graphene universal conductivity $\sigma_{0}=e^{2} / 4 \hbar$. The variables of Table I were used, and a nonradiative decay rate $\eta=20 \mathrm{meV}$ was considered for the interacting (excitonic) calculation.

are considered, the conductivities $\sigma_{+, 1}$ and $\sigma_{-, 1}$ are valley selective, since $\sigma_{+, 1}$ only couples with the $K$ valley $(\tau=1)$, and $\sigma_{-, 1}$ couples with the $K^{\prime}$ valley $(\tau=-1)$.

As was mentioned before, and is visible in Fig. 3, the mean of $\sigma_{+, 1}$ and $\sigma_{-, 1}$ gives us the real part of the longitudinal conductivity. In a similar procedure, and according to Eq. (15), their difference gives access to the imaginary part of the Hall conductivity. In fact, the full expression for $\sigma_{x y}$ is given by

$$
\sigma_{x y}=\left(\frac{\sigma_{+, 2}-\sigma_{-, 2}}{2}\right)+i\left(\frac{\sigma_{-, 1}-\sigma_{+, 1}}{2}\right),
$$

where $\sigma_{ \pm, 1}=2 P_{ \pm} /\left(A E_{0}^{2}\right)$, the imaginary part is the expression presented in Eq. (15), and the real part is readily obtained from a Kramers-Kronig transformation. Both the real and imaginary parts of the Hall conductivity are plotted in Fig. 4. In the same figure, the independent particle approximation is also depicted as thin dashed lines.

The analysis of the data depicted in the figures shows that, curiously enough, when passing from the noninteracting case to the one where excitonic effects are taken into account, an (approximated) inversion of behavior occurs between the real and imaginary parts of $\sigma_{x y}$. If we compare $\sigma_{x y 1}$ with excitons, with $\sigma_{x y 2}$ in the noninteracting case, the former appears to be a smoothed out version of the latter. The same goes for $\sigma_{x y 2}$ with excitons, and $\sigma_{x y 1}$ without them.

The difference between the optical conductivities computed with and without excitonic effects can be understood by taking a closer look at Fig. 3. Looking at the difference between $\sigma_{-, 1}$ and $\sigma_{+, 1}$, we see that, at lower energies, both conductivities are close to zero. Then, $\sigma_{-, 1}$ starts to outgrow $\sigma_{+, 1}$ until the former reaches a maximum, which means that $\sigma_{x y 2}$ also reaches a maximum. Next, $\sigma_{-, 1}$ decreases, and $\sigma_{+, 1}$ increases, which leads to $\sigma_{x y 2}=0$ when the lines intercept, and afterwards to a minimum of $\sigma_{x y 2}$, when $\sigma_{+, 1}$ reaches its maximum. Subsequently, $\sigma_{-, 1}$ decreases, and both conductivities are, again, close to zero. From this point onward

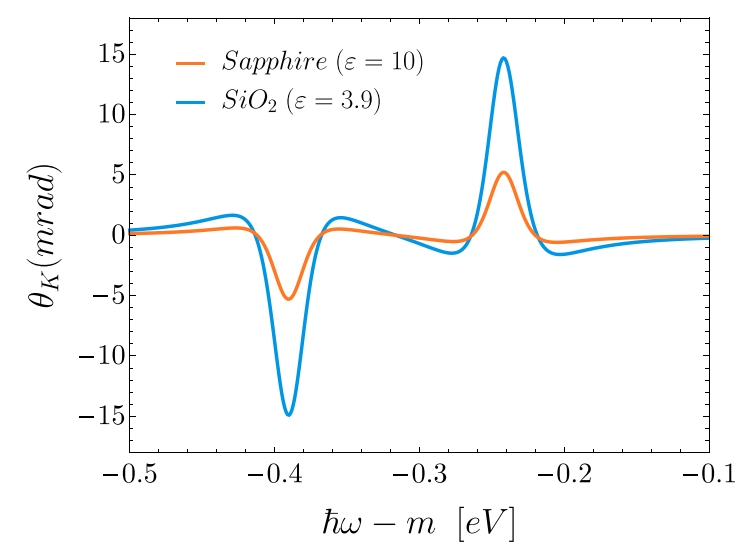

FIG. 5. Representation of the Kerr angle, when excitonic effects are considered, using Eq. (5), and the conductivities previously obtained in Fig. 4. We show the Kerr angle for two substrates, quartz and sapphire, and verify that as the substrate dielectric constant increases, the Kerr angle intensity decreases. Although in principle one should consider the effects of the $\mathrm{hBN}$ layer and cobalt thin film, we consider the substrate contribution as the dominant one, and discard the effect of the other two materials.

the process happens in reverse order. Comparing now this description with Fig. 4, we realize that this is precisely the behavior presented by $\sigma_{x y 2}$.

Now that the conductivities are determined, we can move on to calculating the effect of excitons on the Kerr angle. It can be shown [32] that, in the limit of small angles, the Kerr angle is related to the linear and Hall conductivity through the following equation,

$$
\theta_{K}=\operatorname{Re}\left(\frac{2 c \mu_{0} \sigma_{x y}}{(\varepsilon-1)+\Sigma}\right),
$$

where $\varepsilon$ is the dielectric constant of the substrate, $c$ is the light speed in vacuum, $\mu_{0}$ is the vacuum permittivity,

$$
\Sigma=2 \sqrt{\varepsilon} c \mu_{0} \sigma_{x x}+c^{2} \mu_{0}^{2}\left(\sigma_{x x}^{2}+\sigma_{x y}^{2}\right)+2 i c \mu_{0} \sigma_{x y},
$$

and $\sigma_{x x}$ and $\sigma_{x y}$ are the conductivities previously defined. We should note that in Ref. [33] it has been experimentally shown that even the presence of a single graphene layer is enough to substantially change the exciton binding energies. Here, due to the complexity of the considered apparatus, it is no easy task to give a full description of the effects of the hBN layer, the cobalt thin film, and the substrate on the TMD. We do believe, however, that the effect of the substrate dominates over all others, and thus only consider its contribution to the problem. Using Eq. (19) and the conductivities formerly obtained, we compute the Kerr angle plotted in Fig. 5.

As expected, since $\varepsilon$ is far greater than any other element on the denominator of Eq. (19), the Kerr angle takes its shape from the real part of the Hall conductivity. We can also see that as the environment's dielectric screening increases (when passing to a sapphire substrate), the Kerr angle intensity decreases. Regarding the magnitude, we obtain a Kerr angle that is orders of magnitude higher than the ones from Refs. [34,35], where a 2D electron gas is studied (at $10 \mathrm{~K}$ in the case of Ref. [35]). If the broadening parameter was reduced, then the Kerr angle magnitude would be even larger. 
The $20 \mathrm{meV}$ considered in this paper is a conservative value, and TMDs encapsulated in hBN can have broadenings as low as $2 \mathrm{meV}$ [36].

We also note that in Ref. [37] the Kerr angle for a 4-nmthick cobalt film was measured, presenting a magnitude one order of magnitude smaller when compared to the values of Fig. 5. Therefore, an experiment made in the system proposed in this paper will essentially probe the Kerr effect due to the TMD.

In Ref. [38] the Kerr angle was studied for bilayer $\mathrm{MoS}_{2}$. In this paper, an electric field was applied perpendicularly to the samples, which led to the spatial separation of the $K$ and $K^{\prime}$ valleys. When a spatial map of the Kerr angle was constructed, negative values were observed for the Kerr angle, followed by positive ones, similar to the plot of Fig. 5 .

\section{CONCLUSIONS}

In this paper, we have studied the effects produced on a $\mathrm{MoS}_{2}$ band structure due to proximity to a cobalt ferromagnetic thin film (three layers), more specifically, the case of $\mathrm{MoS}_{2}$ on a heterostructure composed of $\mathrm{MoS}_{2} / \mathrm{hBN} / \mathrm{Co} /$ quartz was studied.

We started using an effective low-energy Hamiltonian, composed of a massive Dirac Hamiltonian, a spin-orbit coupling term, and an exchange contribution, to theoretically describe the changes that the TMD band structure undergoes when placed in the vicinity of a ferromagnetic thin film. We have verified that, in the presence of magnetic proximity effects, the valleys $K$ and $K^{\prime}$ have an asymmetric band structure, leading to different interactions with the two components of circularly polarized light.

Using Fermi's golden rule, we then proceeded to the computation of the longitudinal conductivity $\sigma_{x x}$, and Hall conductivity $\sigma_{x y}$ in the noninteracting limit (the results can be found in the Supplemental Material [30]). The existence of a finite Hall conductivity is a direct consequence of the asymmetry between the $K$ and $K^{\prime}$ valleys produced by proximity effects. Afterwards, we presented a semianalytical method that allowed us to extend our work in the noninteracting limit to the case where excitons are present. Using this method, we once more computed the optical conductivities, but now taking into consideration the excitonic effects. Once again, we obtained a nonzero Hall conductivity due to the different interactions of the two components of circularly polarized light with the TMD. An intriguing finding appeared when the plots of the Hall conductivity in the noninteracting and in the interacting limits were compared. Looking at Fig. 4, it is possible to see that an inversion of behavior between the real and imaginary parts of the Hall conductivity takes place when passing between these two limits, that is, if we compare $\sigma_{x y 1}$ with excitons, with $\sigma_{x y 2}$ in the noninteracting case, the former appears to be a smoothed out version of the latter. The same happens for $\sigma_{x y 2}$ with excitons and $\sigma_{x y 1}$ without them. This inversion is a direct consequence of the dramatic change of the line shape of the optical conductivity when excitonic effects are included.

Finally, we used the Hall conductivity containing excitonic effects to obtain the Kerr rotation angle. Since the Kerr rotation angle has a direct dependence on $\sigma_{x y}$, it can only be explored when an asymmetry between the valleys is induced, that is, when the TMD is proximitized.

\section{ACKNOWLEDGMENTS}

N.M.R.P. acknowledges support from the European Commission through the project "Graphene-Driven Revolutions in ICT and Beyond" (Ref. No. 785219), and the Portuguese Foundation for Science and Technology (FCT) in the framework of the Strategic Financing UID/FIS/04650/2019. In addition, N.M.R.P. acknowledges COMPETE2020, PORTUGAL2020, FEDER, and the Portuguese Foundation for Science and Technology (FCT) through Projects No. PTDC/FIS-NAN/3668/2013, No. POCI-01-0145-FEDER-028114, No. POCI-01-0145FEDER-029265, No. PTDC/NAN-OPT/29265/2017, and No. POCI-01-0145-FEDER-02888. G.C. acknowledges Fundação para a Ciência e a Tecnologia (FCT) for Grant No. SFRH/BD/138806/2018. G.C. and J.F.-R. acknowledge financial support from FCT through Grant No. P2020-PTDC/FIS-NAN/4662/2014. J.F.-R. acknowledges financial support from FCT for the P2020-PTDC/FISNAN/4662/2014, P2020-PTDC/FIS-NAN/3668/2014, and the UTAPEXPL/NTec/0046/2017 projects, as well as Generalitat Valenciana funding Prometeo2017/139 and MINECO Spain (Grant No. MAT2016-78625-C2).

\section{APPENDIX: FROM BSE TO THE WANNIER EQUATION AND ITS SOLUTION}

In second quantization, the state of an exciton of momentum $\mathbf{Q}$, in motion in a TMD monolayer of area $A$, can be written as

$$
|v, \mathbf{Q}\rangle=\frac{1}{\sqrt{A}} \sum_{\mathbf{k}} \phi_{v}(\mathbf{k}) a_{\mathbf{k}+\mathbf{Q}, c}^{\dagger} a_{\mathbf{k}, v}|\mathrm{GS}\rangle
$$

where the state $|\mathrm{GS}\rangle$ represents the electronic ground state of the TMD, that is, a filled valence band and an empty conduction band; $\phi_{v}(\mathbf{k})$ is the Fourier transform of the real-space exciton wave function, with $v$ representing the principal and magnetic quantum numbers that characterize it; the second quantized operators $a_{\mathbf{k}+\mathbf{Q}, c}^{\dagger}$ and $a_{\mathbf{k}, v}$ create and annihilate an electron of momentum $\mathbf{k}+\mathbf{Q}$ in the conduction band and an electron of momentum $\mathbf{k}$ in the valence band, respectively. This state can be expressed in condensed form as $|v, \mathbf{Q}\rangle=$ $b_{\mathbf{Q}, v}^{\dagger}|\mathrm{GS}\rangle$, with $\mathbf{b}_{\mathbf{Q}, v}^{\dagger}$ the bosonic operator, defined as

$$
b_{\mathbf{Q}, v}^{\dagger}=\frac{1}{\sqrt{A}} \sum_{\mathbf{k}} \phi_{\nu}(\mathbf{k}) a_{\mathbf{k}+\mathbf{Q}, c}^{\dagger} a_{\mathbf{k}, v} .
$$

We note that the bosonic nature of the operator (A2) is guaranteed only in an average over the ground state.

The electrons in the TMD monolayer are described by the Hamiltonian $H=H_{0}+V$, where

$$
H_{0}=\sum_{\lambda, \mathbf{k}} E_{\lambda, \mathbf{k}} \hat{a}_{\lambda, \mathbf{k}}^{\dagger} \hat{a}_{\lambda, \mathbf{k}}
$$


with $\lambda=\{c, v\}, E_{\lambda, \mathbf{k}}=E_{\alpha}$, and with the interaction term given by

$$
\begin{aligned}
V= & \frac{1}{2 A} \sum_{\mathbf{k}_{1}, \mathbf{k}_{2}, \mathbf{p}} \sum_{\lambda_{1} \lambda_{2} \lambda_{3} \lambda_{4}} V(\mathbf{p}) F_{\lambda_{1} \lambda_{2} \lambda_{3} \lambda_{4}}\left(\mathbf{k}_{1}, \mathbf{k}_{2}, \mathbf{p}\right) \\
& \times \hat{a}_{\mathbf{k}_{1}+\mathbf{p}, \lambda_{1}}^{\dagger} \hat{a}_{\mathbf{k}_{2}-\mathbf{p}, \lambda_{2}}^{\dagger} \hat{a}_{\mathbf{k}_{2}, \lambda_{3}} \hat{a}_{\mathbf{k}_{1}, \lambda_{4}},
\end{aligned}
$$

and

$$
F_{\lambda_{1} \lambda_{2} \lambda_{3} \lambda_{4}}\left(\mathbf{k}_{1}, \mathbf{k}_{2}, \mathbf{p}\right)=u_{\mathbf{k}_{1}+\mathbf{p}, \lambda_{1}}^{\dagger} u_{\mathbf{k}_{2}-\mathbf{p}, \lambda_{2}}^{\dagger} u_{\mathbf{k}_{2}, \lambda_{3}} u_{\mathbf{k}_{1}, \lambda_{4}},
$$

is a product of the spinors presented in Eqs. (2) and (3) from the main text and is termed the form factor. It is important to note that we do not specify the spin and valley indices of the spinors, since the procedure is identical for any spin and valley combination. The function $V(\mathbf{p})$ is the Fourier transform of the Rytova-Keldysh potential given in Eq. (5), and whose expression is known analytically [28],

$$
V(\mathbf{q})=\frac{e^{2}}{2 \epsilon_{0} q\left(r_{0} q+\kappa\right)} .
$$

We now intend to show that if the state $|\nu, \mathbf{Q}\rangle$ is an eigenstate of $H$, then $\phi_{\nu}(\mathbf{k})$ obeys the Bethe-Salpeter equation, which when Fourier transformed to the real space becomes the Wannier equation, under a set of simplifying assumptions.

As previously said, with the purpose of obtaining the Bethe-Salpeter equation, we start assuming that the state (A1) is an eigenstate of the Hamiltonian $H$. If this is true, then $H$ can be written as $H=\sum_{\mathbf{Q}, v} E_{\mathbf{Q}, v} b_{\mathbf{Q}, v}^{\dagger} b_{\mathbf{Q}, v}$, where $E_{\mathbf{Q}, v}$ are the energy eigenvalues of the exciton. Afterwards, we evaluate the commutator of $H$ with $b_{\mathbf{Q}, v}^{\dagger}$ using both the fermionic (when dealing with the $a$ and $a^{\dagger}$ operators) and bosonic (when dealing directly with the $b$ and $b^{\dagger}$ operators) representations. In the end we demand that both results must be equal. Following this procedure, one obtains the equation for the wave function of the exciton in momentum space,

$$
\begin{aligned}
E \phi_{v}(\mathbf{k})= & \phi_{v}(\mathbf{k})\left(E_{c, \mathbf{k}}-E_{v, \mathbf{k}}\right)+\frac{1}{A} \phi_{v}(\mathbf{k}) \sum_{\mathbf{p}} V(\mathbf{p}) \\
& \times\left[u_{\mathbf{k}, v}^{\dagger} u_{\mathbf{k}-\mathbf{p}, v}^{\dagger} u_{\mathbf{k}, v} u_{\mathbf{k}-\mathbf{p}, v}-u_{\mathbf{k}, c}^{\dagger} u_{\mathbf{k}+\mathbf{p}, v}^{\dagger} u_{\mathbf{k}, c} u_{\mathbf{k}+\mathbf{p}, v}\right] \\
& -\frac{1}{A} \sum_{\mathbf{p}} V(\mathbf{p}) \phi_{v}(\mathbf{p}+\mathbf{k}) u_{\mathbf{p}+\mathbf{k}, v}^{\dagger} u_{\mathbf{k}, c}^{\dagger} u_{\mathbf{p}+\mathbf{k}, c} u_{\mathbf{k}, v},
\end{aligned}
$$

where $E$ represents the exciton energy eigenvalues. This equation in momentum space for $\phi_{v}(\mathbf{k})$ is known as the BetheSalpeter equation. Analyzing it, one realizes that each term has a clear and distinct meaning. While the first term gives the energy of a particle-hole excitation when no interactions are treated, the second term represents the exchange energy correction to the noninteracting particle-hole excitation energy; its value determines the magnitude of the gap. The third and final term describes the attraction between the electron and hole present in the conduction and in the valence band, respectively. Crucially, this term is negative, although in the original Hamiltonian the interaction between electrons is obviously repulsive. To solve this equation directly in the momentum space, one would have to solve an integral equation, that, although possible, can be a rather delicate process [8].
Another way of solving this problem passes by converting this integral equation into a differential one, going to real space by means of a Fourier transform. Unfortunately, another complication arises, since the spinor product in the third term of the Bethe-Salpeter equation makes Fourier transforming an almost impossible task. In order to solve this problem, we make the following observation concerning the form factors. In the case of a large energy gap, one can take

$$
u_{\mathbf{p}+\mathbf{k}, v}^{\dagger} u_{\mathbf{k}, c}^{\dagger} u_{\mathbf{p}+\mathbf{k}, c} u_{\mathbf{k}, v} \longrightarrow 1+O\left(1 / m^{2}\right)
$$

so as to forego the spinorial structure of the last term of the Bethe-Salpeter equation. Although the reader, recalling the values of Table I, may find this approximation to be crude, we actually found excellent agreement with the results from Ref. [26] when the case of $\mathrm{MoTe}_{2}$ on a substrate of $\mathrm{EuO}$ was studied. It is also considered that both the energy difference $E_{c, \mathbf{k}}-E_{v, \mathbf{k}}$ and the exchange energy corrections are expanded up to second order in $\mathbf{k}$. The resulting differential equationin real space—reads

$$
\left(E-E_{g}\right) \psi_{v}(\mathbf{r})=-\left[\frac{\hbar^{2}}{2 \mu} \nabla^{2}+V(\mathbf{r})\right] \psi_{\nu}(\mathbf{r}),
$$

which is known as the Wannier equation for the excitonic wave function. In this context, $\mu$ is the reduced mass of the exciton which, in our model, reads $m^{*} / 2$, with $m^{*}$ the effective mass of the electron/hole, and $E_{g}$ is the corrected gap considering the exchange correction. In this work, however, for simplicity's sake, we will discard the exchange correction, and consider $E_{g}$ as the gap given by Eq. (4) of the main text. This decision allows us to better compare our results with the ones from Ref. [26]. If the exchange correction to the gap was considered, the separation between the valence and conduction bands would increase, the conductivities would appear at higher energies, and their magnitude would be bigger.

In the above we have shown that in order to solve the excitonic problem, one needs to first obtain the excitonic wave function in both real and reciprocal space. Here, we show that a quasianalytical expression for the wave functions of the exciton can be written using a set of Gaussian functions, which simplifies the calculations when compared to a fully numerical method. Although other possibilities exist for the choice of a basis, such as the Slater basis, the choice of a Gaussian basis is the approach used in this work. Inspired by the solution of the 2D hydrogen atom [39], we write our wave function as

$$
\psi_{v}(\mathbf{r})=\mathcal{A}_{v} \sum_{j=1}^{N} c_{j}^{v} e^{i m \theta} r^{|m|} e^{-\zeta_{j} r^{2}},
$$

where $e^{i m \theta} r^{|m|}$ follows from the eigenfunctions of the $z$ component of the angular momentum and the behavior of the radial wave function near the origin, for $m=0, \pm 1, \pm 2, \ldots$, the magnetic quantum number; the Gaussian term $e^{-\zeta_{j} r^{2}}$ describes the decay of the wave function far from the origin, with a decay constant dependent on $\zeta_{j}$. The coefficients $c_{j}^{v}$ weigh the different terms; and $\mathcal{A}_{v}$ is a normalization constant given by

$$
\mathcal{A}_{v}=\sqrt{\frac{1}{\pi \mathcal{S}_{v}}}
$$


with $\mathcal{S}_{v}=\sum_{j=1}^{N} \sum_{j^{\prime}=1}^{N} c_{j}^{\nu *} c_{j^{\prime}}^{\nu}\left(\zeta_{j}+\zeta_{j^{\prime}}\right)^{-1-|m|} \Gamma(|m|+1)$, and $\Gamma(x)$ the gamma function. An additional advantage of this method is that the matrix elements of both the kinetic operator and the electron-electron interaction do not mix different $m$ values and therefore the eigenvalue problem is block diagonal in the angular momentum space.

Using our trial wave function and computing the matrix elements of the kinetic and potential energy operators, the generalized eigenvalue problem acquires the form

$$
\sum_{j=1}^{N}\left[H\left(\zeta_{i}, \zeta_{j}\right)-S\left(\zeta_{i}, \zeta_{j}\right) E\right] c_{j}^{\nu}=0
$$

where $H\left(\zeta_{i}, \zeta_{j}\right)$ is called the Hamiltonian kernel and $S\left(\zeta_{i}, \zeta_{j}\right)$ is the superposition kernel. The latter differs from a Kronecker- $\delta$ kernel, since the set of Gaussian functions is not an orthogonal basis. Both kernels have an analytical expression given in Ref. [31]. Equation (A12) has first been written in nuclear physics and is termed the Griffin-Hill-Wheeler equation [40]. The key aspect of the method is the sensible choice of the parameters $\zeta_{j}$. A choice not so well known is the use of a logarithmic grid of $\zeta$ 's according to the rule given in Ref. [41], $\Omega=\frac{\ln \zeta}{A}$, where $A>1$ and the $\Omega$ 's are uniformly spaced in an interval $\left[\Omega_{\min }, \Omega_{\max }\right]$ and $A$ is typically chosen between 6 and 8 . The exposed method was previously used in Ref. [31], and was shown to produce excellent results.
[1] R. Holm and W. Meissner, Z. Phys. 74, 715 (1932).

[2] K. Novoselov, A. Mishchenko, A. Carvalho, and A. C. Neto, Science 353, aac9439 (2016).

[3] A. I. Buzdin, Rev. Mod. Phys. 77, 935 (2005).

[4] I. Žutić, A. Matos-Abiague, B. Scharf, H. Dery, and K. Belashchenko, Mater. Today 22, 85 (2019).

[5] Z. Wang, C. Tang, R. Sachs, Y. Barlas, and J. Shi, Phys. Rev. Lett. 114, 016603 (2015).

[6] L. Fu and C. L. Kane, Phys. Rev. Lett. 100, 096407 (2008).

[7] G. Wang, A. Chernikov, M. M. Glazov, T. F. Heinz, X. Marie, T. Amand, and B. Urbaszek, Rev. Mod. Phys. 90, 021001 (2018).

[8] A. J. Chaves, R. M. Ribeiro, T. Frederico, and N. M. R. Peres, 2D Mater. 4, 025086 (2017).

[9] D. Xiao, G.-B. Liu, W. Feng, X. Xu, and W. Yao, Phys. Rev. Lett. 108, 196802 (2012).

[10] D. MacNeill, C. Heikes, K. F. Mak, Z. Anderson, A. Kormányos, V. Zólyomi, J. Park, and D. C. Ralph, Phys. Rev. Lett. 114, 037401 (2015).

[11] A. V. Stier, K. M. McCreary, B. T. Jonker, J. Kono, and S. A. Crooker, Nat. Commun. 7, 10643 (2016).

[12] A. Arora, R. Schmidt, R. Schneider, M. R. Molas, I. Breslavetz, M. Potemski, and R. Bratschitsch, Nano Lett. 16, 3624 (2016).

[13] C. Zhao, T. Norden, P. Zhang, P. Zhao, Y. Cheng, F. Sun, J. P. Parry, P. Taheri, J. Wang, Y. Yang, T. Scrace, K. Kang, S. Yang, G. xing Miao, R. Sabirianov, G. Kioseoglou, W. Huang, A. Petrou, and H. Zeng, Nat. Nanotechnol. 12, 757 (2017).

[14] D. Zhong, K. L. Seyler, X. Linpeng, R. Cheng, N. Sivadas, B. Huang, E. Schmidgall, T. Taniguchi, K. Watanabe, M. A. McGuire et al., Sci. Adv. 3, e1603113 (2017).

[15] K. L. Seyler, D. Zhong, B. Huang, X. Linpeng, N. P. Wilson, T. Taniguchi, K. Watanabe, W. Yao, D. Xiao, M. A. McGuire et al., Nano Lett. 18, 3823 (2018).

[16] J. Qi, X. Li, Q. Niu, and J. Feng, Phys. Rev. B 92, 121403 (2015)

[17] L. Xu, M. Yang, L. Shen, J. Zhou, T. Zhu, and Y. P. Feng, Phys. Rev. B 97, 041405(R) (2018).
[18] N. Li, J. Zhang, Y. Xue, T. Zhou, and Z. Yang, Phys. Chem. Chem. Phys. 20, 3805 (2018).

[19] Q. Zhang, S. A. Yang, W. Mi, Y. Cheng, and U. Schwingenschlögl, Adv. Mater. 28, 959 (2016).

[20] K. Zollner, P. E. Faria Junior, and J. Fabian, Phys. Rev. B 100, 085128 (2019).

[21] K. Zollner, P. E. Faria Junior, and J. Fabian, arXiv:1910.13223.

[22] A. Cebollada, D. Weller, J. Sticht, G. R. Harp, R. F. C. Farrow, R. F. Marks, R. Savoy, and J. C. Scott, Phys. Rev. B 50, 3419 (1994).

[23] W. Zeper, F. Greidanus, P. Carcia, and C. Fincher, J. Appl. Phys. 65, 4971 (1989).

[24] P. M. Oppeneer, T. Maurer, J. Sticht, and J. Kübler, Phys. Rev. B 45, 10924 (1992).

[25] H. Da, L. Gao, W. Ding, and X. Yan, J. Phys. Chem. Lett. 8, 3805 (2017).

[26] B. Scharf, G. Xu, A. Matos-Abiague, and I. Žutić, Phys. Rev. Lett. 119, 127403 (2017).

[27] L. V. Keldysh, Sov. J. Exp. Theor. Phys. Lett. 29, 658 (1979).

[28] S. Rytova, Moscow Univ. Phys. Bull. 22, 30 (1967).

[29] A. Kormányos, G. Burkard, M. Gmitra, J. Fabian, V. Zólyomi, N. D. Drummond, and V. Fal'ko, 2D Mater. 2, 022001 (2015).

[30] See Supplemental Material at http://link.aps.org/supplemental/ 10.1103/PhysRevB.101.045408 for the calculations for the noninteracting case, as referred to in the main text.

[31] J. C. G. Henriques, G. B. Ventura, C. D. M. Fernandes, and N. M. R. Peres, J. Phys.: Condens. Matter 32, 025304 (2019).

[32] G. Catarina, N. M. R. Peres, and J. Fernández-Rossier, arXiv:1910.13371.

[33] A. Raja, A. Chaves, J. Yu, G. Arefe, H. M. Hill, A. F. Rigosi, T. C. Berkelbach, P. Nagler, C. Schüller, T. Korn, C. Nuckolls, J. Hone, L. E. Brus, T. F. Heinz, D. R. Reichman, and A. Chernikov, Nat. Commun. 8, 15251 (2017).

[34] A. A. Perov, L. V. Solnyshkova, and D. V. Khomitsky, Phys. Rev. B 82, 165328 (2010).

[35] C. L. Yang, H. T. He, L. Ding, L. J. Cui, Y. P. Zeng, J. N. Wang, and W. K. Ge, Phys. Rev. Lett. 96, 186605 (2006). 
[36] F. Cadiz, E. Courtade, C. Robert, G. Wang, Y. Shen, H. Cai, T. Taniguchi, K. Watanabe, H. Carrere, D. Lagarde, M. Manca, T. Amand, P. Renucci, S. Tongay, X. Marie, and B. Urbaszek, Phys. Rev. X 7, 021026 (2017).

[37] S. M. Hamidi, M. A. Oskuei, S. Sadeghi, and M. M. Tehranchi, J. Supercond. Novel Magn. 27, 867 (2014).
[38] J. Lee, K. F. Mak, and J. Shan, Nat. Nanotechnol. 11, 421 (2016).

[39] X. L. Yang, S. H. Guo, F. T. Chan, K. W. Wong, and W. Y. Ching, Phys. Rev. A 43, 1186 (1991).

[40] J. J. Griffin and J. A. Wheeler, Phys. Rev. 108, 311 (1957).

[41] J. R. Mohallem, Z. Phys. D: At., Mol. Clusters 3, 339 (1986). 